\title{
Influence of expansion rate on circumferential residual stress distribution of expandable casing
}

\author{
Zhang Jianbing $^{1 *}$, Kong Detao ${ }^{1}$, Wang Qian ${ }^{1}$, Wang Haobo ${ }^{1}$ and Wang Haiying ${ }^{1}$ \\ ${ }^{1}$ Mechanical Engineering College, Xi'an Shiyou University, Xi'an, Shaanxi, 710065, China
}

\begin{abstract}
To study the distribution law of circumferential residual stress after casing expansion, using the finite element explicit dynamic analysis method analyzed the expansion process of expandable casings under different expansion rates. The analysis obtained key technical parameters of circumferential residual stress, average circumferential residual stress and elastic recovery along the wall thickness direction after casing expansion. It is recognized that the maximum residual tensile stress after casing expansion locates in the middle part of the casing thickness direction. The maximum residual compressive stress locates in the outer wall of the casing. When the expansion rate exceeds $18 \%$, the increase in expansion rate will not lead to an increase in circumferential residual stress after casing expansion. The elastic recovery after casing expansion will reduce the circumferential stress during the expansion process. Considering collapse strength and the influence of elastic recovery on casing patch sealing performance after casing expansion, $23 \%$ is the most suitable expansion rate, which can effectively reduce the circumferential residual stress and improve the casing collapse strength. The analysis in this paper can provide bases for the calculation of casing collapse strength after expansion.
\end{abstract}

\section{Introduction}

Expandable casing technology is a major new technology in petroleum engineering in recent years, which is widely used in drilling, completion, oil production, workover and other fields. This technology can expand the casing diameter in the wellbore, thus saving the wellbore size and the cost of well construction. Through the previous analysis and test, the collapse strength of casing after expansion will decrease to $40 \%-50 \%$ of the casing strength before expansion, which has a great influence on the safety and reliability of the underground casing column. Casing expansion generates residual stress, which circumferential residual stress has the greatest impact on the collapse strength after casing expansion.

S.Al-Hiddab et al. ${ }^{[1-2]}$ studied the effect of equivalent stress and residual stress on the expansion process, the distribution and condition of residual stress after casing expansion. Kiptisia W et al. ${ }^{[3-5]}$ used theoretical analysis and finite element method to analyze the residual stress of tube transition zone and joint. Xu Binggui et al. ${ }^{6}$ proposed the application of stress, strain and residual stress of solid expansion pipe in the process of change to repair casing. Zhang Jianbing et al. $^{7}$ measured and analyzed the transverse residual stress of the casing after expansion by ring cutting method. Tang Ming ${ }^{[8-9]}$ proposed different directions of residual stress on the surface of the expansion casing and the outer cylinder, and measured the axial and circumferential residual stress of the inner and outer walls, the weld fusion zone and the heat-affected area before and after expansion. Tang Hailiang ${ }^{10}$ used finite element software to analyze the influence of different yield strengths on the residual stress of the expansion tube.

The residual stress in the process of casing expansion is uneven in distribution ${ }^{[11-12]}$. It is tough and expensive to measure the residual stress law by experiments. Finite element simulation can solve this problem efficiently. In this paper, using the finite element software analyzed that the distribution of circumferential residual stress along the wall thickness direction under different expansion rates and the influence of elastic recovery on residual stress after casing expansion, which provides bases for calculating the collapse strength after casing expansion.

\section{Finite element model and condition settings}

In this paper, using the finite element analysis software Workbench LS-DYNA module simulates the expansion process of the expandable casing. The physical model will be explained in figure 1 . The inner diameter of the expandable casing is $\Phi 122.7 \mathrm{~mm}$. The outer diameters of the expansion cone are $138.7 \mathrm{~mm}, 144.8 \mathrm{~mm}, 150.9 \mathrm{~mm}$ and $157.2 \mathrm{~mm}$, respectively. The corresponding casing expansion rates are $13 \%, 18 \%, 23 \%$ and $28 \%$, respectively.

\footnotetext{
*Corresponding author's e-mail: zhjb@xsyu.edu.cn
} 


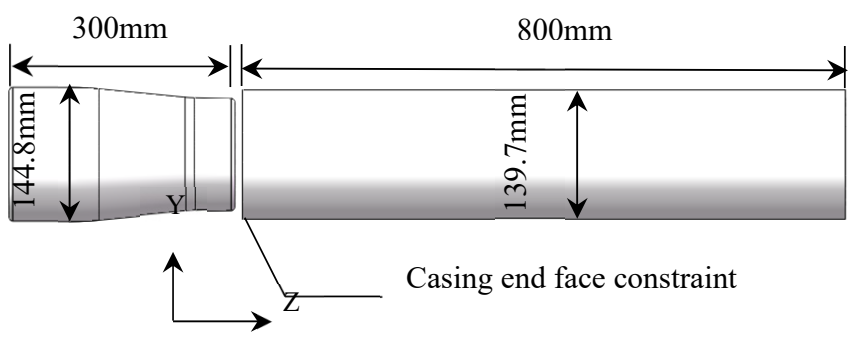

Figure 1. Expandable casing physical model.

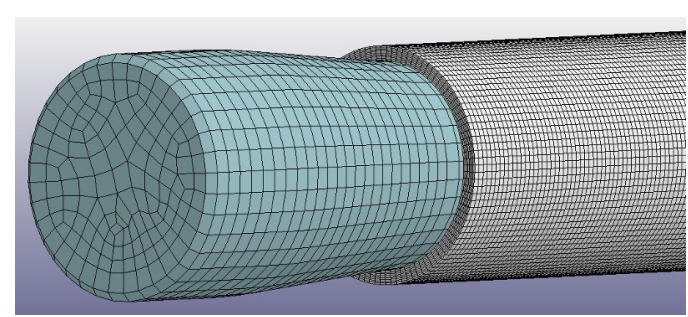

Figure 2. The meshing of expansion model.
In the finite element analysis setting, the expandable casing is set as a flexible body, and the expansion cone is set as a rigid body. The material which is set as a bilinear kinematic hardening model is suitable for expansion. The expansion cone moves along the $\mathrm{Z}$ direction at a constant speed to simulate the expansion process. The contact

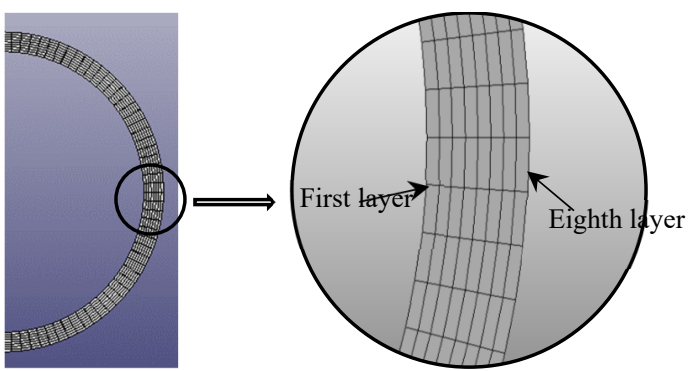

Figure 3. Casing mesh control.

\section{Results of residual stress analysis}

\subsection{Circumferential residual stress}

The circumferential residual stress has the greatest influence on the collapse strength after casing expansion ${ }^{13}$. After the expansion process, three different radial paths are defined at different casing positions, as shown in figure 4. Each path extends from the inner wall to the outer wall of the casing. The circumferential residual stress of each layer of the element is plotted in the same chart, to compare the distribution law of circumferential residual stress at different positions.

The distribution of circumferential residual stress under different paths will be explained in figure 5 when the expansion rate is $18 \%$. The variation trend of circumferential stress from the inner wall to the outer wall of the casing is roughly the same. The residual tensile between expansion cone and casing adopts body-to-body.

The overall mesh of the expansion model will be shown in figure 2 . When dividing mesh, grid layers along the thickness direction of the casing are set as eight layers, which is shown in figure 3 .

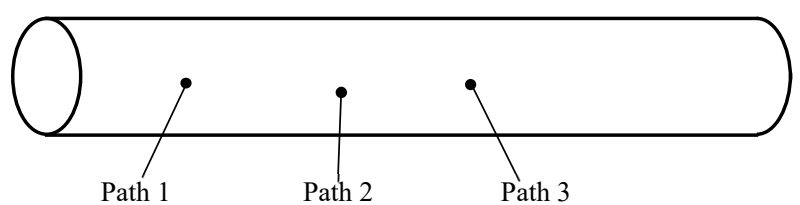

Figure 4. Relative casing position of different paths.

stress is near the inner wall of the casing. The residual compressive stress is near the outer wall of the casing. The maximum residual tensile stress and residual compressive stress of path 1 after expansion are $190.87 \mathrm{MPa}$ and 220.59 $\mathrm{MPa}$. The maximum residual tensile stress and compressive stress of path 2 are 226.08 $\mathrm{MPa}$ and 225.28 $\mathrm{MPa}$. The maximum residual tensile stress and compressive stress of path 3 are 266.33 MPa and 199.33 $\mathrm{MPa}$. From the inner wall to the middle layer of the casing, the residual tensile stress gradually increases and reaches the maximum value in the fourth layer. Then, the residual tensile stress decreases gradually and becomes residual compressive stress between the 5th layer and the 6th layer. The residual compressive stress reaches the maximum value at the outer wall of the casing. Path 1 is the closest to the end face of the casing, and the stress of the inner wall is residual compressive stress. The maximum residual tensile stress of path 1 is smaller than the other two paths because of uneven plastic deformation of the metal during the expansion process. 


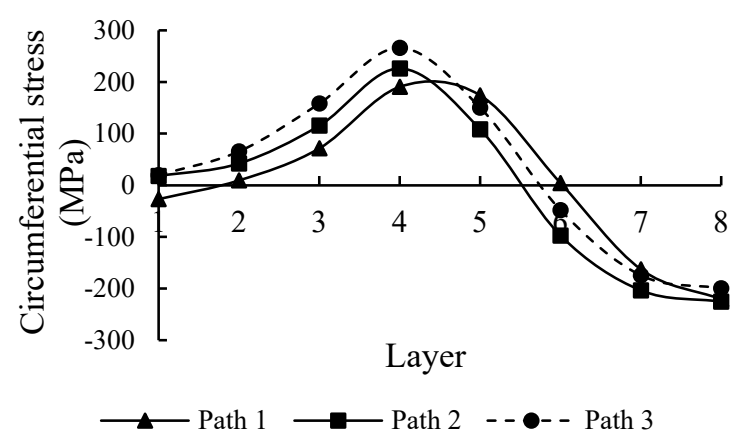

Figure 5. Distribution of circumferential residual stress in the expansion rate of $18 \%$.

The circumferential residual stress distribution will be shown in figure 6 under different expansion rates. When the casing expansion rates are $13 \%, 18 \%$ and $23 \%$, the maximum residual tensile stress locates in the fourth layer, and the maximum residual compressive stress locates in the outer wall of the casing. The residual tensile stress

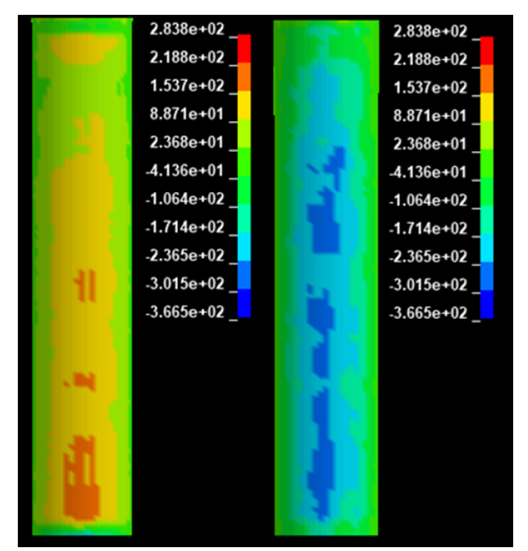

Figure 7. The circumferential residual stress nephogram of $13 \%$ expansion rates.

The circumferential residual stress nephograms will be shown in figure 7 and figure 8 when the expansion rates are $13 \%$ and 18 . Under four different expansion rates, the maximum residual tensile stress values are $283.8 \mathrm{MPa}$, $335.5 \mathrm{MPa}, 287.8 \mathrm{MPa}$ and $278.8 \mathrm{MPa}$, respectively. The maximum residual compressive stress values are 365.5MPa, 284.1MPa, 308.6MPa and 304.9MPa, respectively.

It shows the relation curve between the maximum residual tensile stress and the maximum residual compressive stress under different expansion rates in figure 9 . When the expansion rate is $13 \%$, the maximum

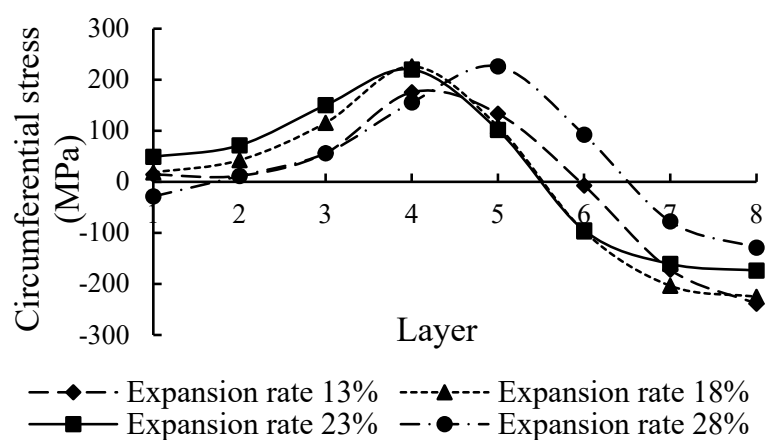

Figure 6. The circumferential stress distribution under different expansion rates.

transforms into residual compressive stress between the fifth layer and the sixth layer. When the expansion rate is $28 \%$, the inner wall of the casing is residual compressive stress. The residual tensile stress changes into residual compressive stress between the sixth layer and the seventh layer.

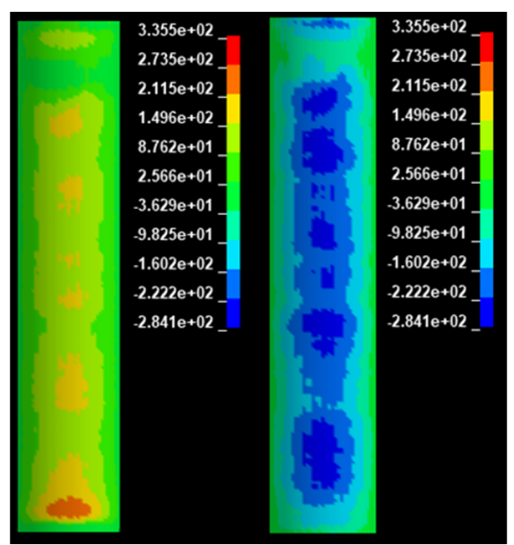

Figure 8. The circumferential residual stress nephogram of $18 \%$ expansion rates.

residual compressive stress is the largest. When the expansion rate is $18 \%$, the maximum residual tensile stress is the largest. There is no linear relationship between the maximum circumferential stress and the expansion rate. The maximum circumferential stress does not change significantly when the expansion rate exceeds $23 \%$. It can be seen from the curve that there is a special relationship between the maximum tensile stress and the maximum compressive stress. If the residual tensile stress increases, the residual compressive stress decreases relatively, conversely the same. 


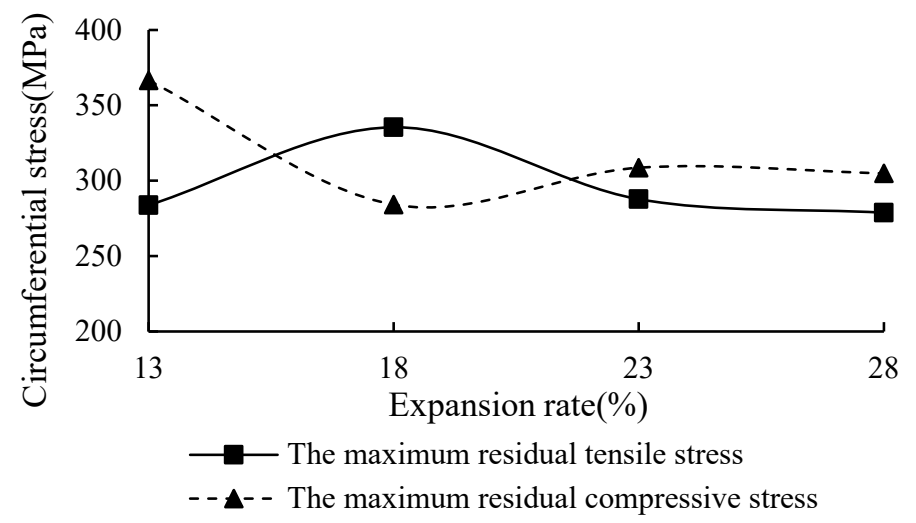

Figure 9. The maximum circumferential stress under different expansion rates.

\subsection{Circumferential average residual stress}

It can be found from figure 7 and figure 8 that the circumferential residual stress after casing expansion is uneven along the axial direction of the casing. The position of the three paths is random and cannot reflect the circumferential residual stress law of the whole casing. It

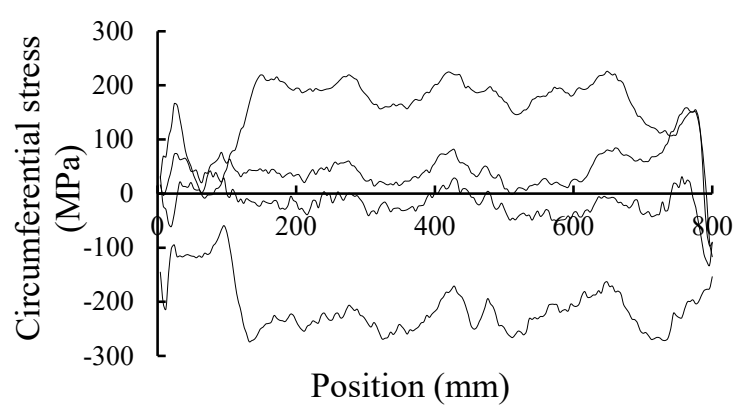

Figure 10. Distribution of circumferential stress along casing axial direction.

The circumferential average stress distribution will be shown in figure 11 under different expansion rates. The maximum circumferential average tensile stress locates in the fifth layer element. The position that average tensile stress transforms into average compressive stress is between the sixth layer and the seventh layer element of the casing, which is closer to the outer wall of the casing.

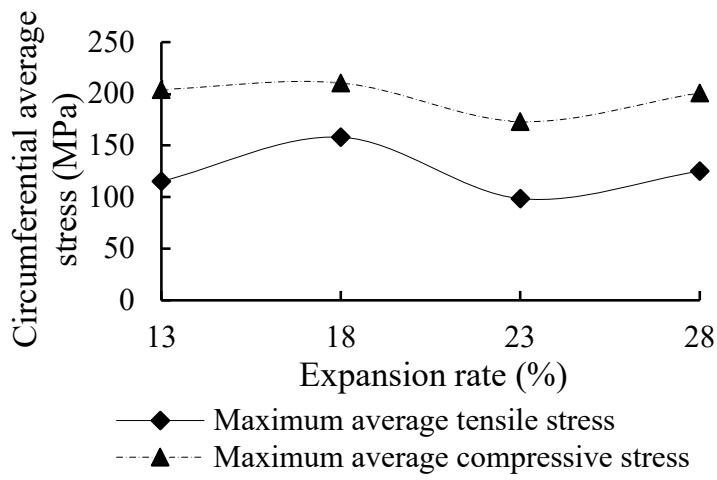

Figure 12. Maximum circumferential average stress under different expansion rates. shows the circumferential stress distribution of the first layer, the third layer, the fifth layer and the eighth layer elements along the casing axis after casing expansion at the expansion rate of $18 \%$ in figure 10 . It can be seen that there are both residual tensile stress and residual compressive stress in the inner wall of the casing. The circumferential stress mutates at both ends of the casing at each layer, with a large variation amplitude.

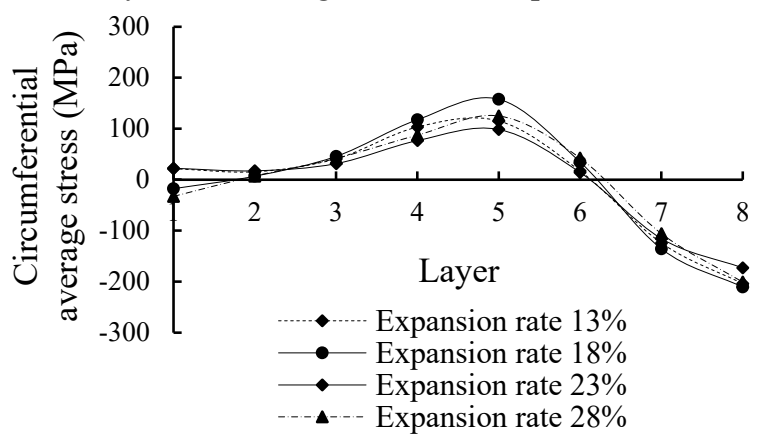

Figure 11. Circumferential average stress distribution under different expansion rates.

The maximum circumferential average compressive stress still locates in the outer wall of the casing. When the expansion rate is $18 \%$ or $28 \%$, the inner wall of the casing is residual compressive stress. Adversely, the inner wall of the casing is residual tensile stress when the expansion rate is $13 \%$ or $23 \%$.

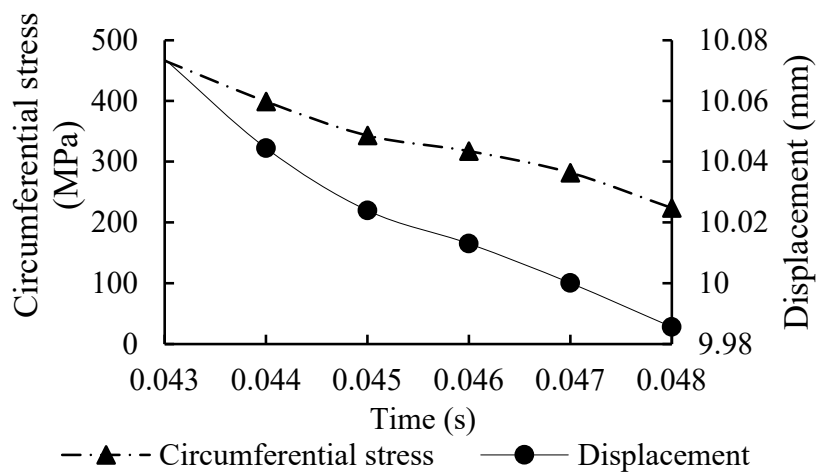

Figure 13. The displacement and circumferential stress variation curves of a unit during $0.043 \sim 0.048 \mathrm{~s}$. 
It shows the maximum circumferential average tensile stress and compressive stress curves under different expansion rates in figure 12 . It can be seen from the figure that when the expansion rate is $18 \%$, the circumferential average tensile stress and compressive stress are the largest, which are 157.9 $\mathrm{MPa}$ and $210.5 \mathrm{MPa}$, respectively. When the expansion rate is $23 \%$, the circumferential average tensile stress and compressive stress are the smallest, which are 98.5 MPa and 173.1 MPa, respectively. Comparing with the two rates, the expansion rate of $23 \%$ can not only expand the wellbore size but also reduce the circumferential residual stress after expansion to improve the collapse strength of the casing. When the expansion rate exceeds $18 \%$, the increase in expansion rate will not lead to an increase in residual stress after casing expansion.

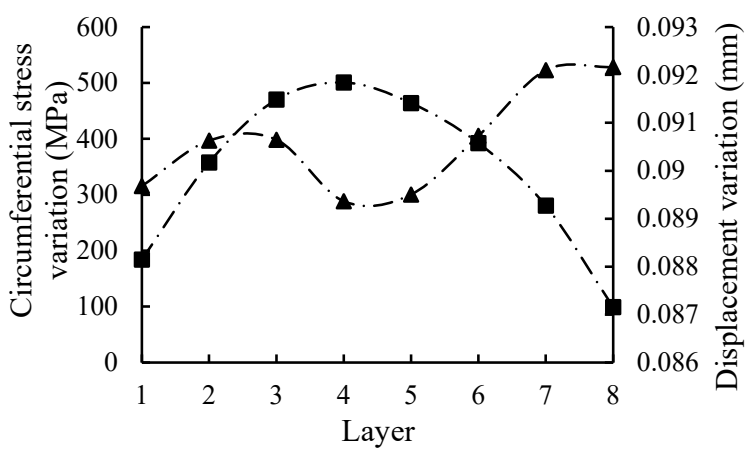

- $\bullet \cdot-$ Circumferential stress variation

- - - Elastic recovery

Figure 14. Elastic recovery and circumferential stress variation of $13 \%$ expansion rate.

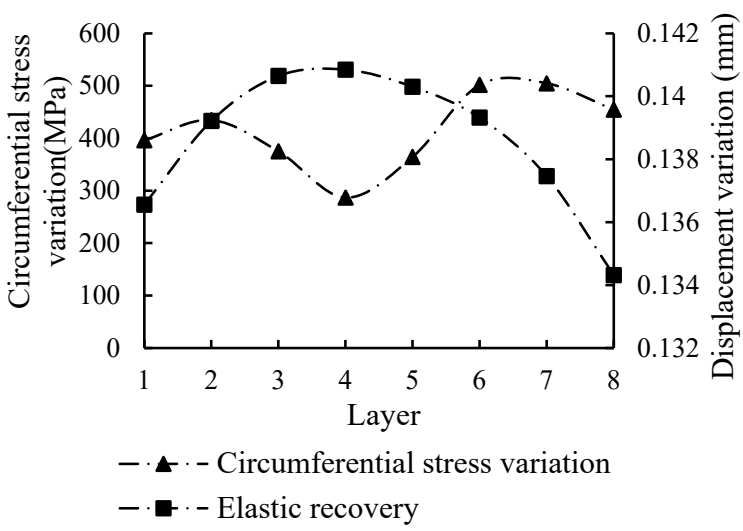

Figure 16. Elastic recovery and circumferential stress variation of $23 \%$ expansion rate.

Comparing the circumferential stress of the casing after expansion and expanding, the radial displacement variation of the casing after the expansion regards as the elastic recovery. It shows the relationship between casing elastic recovery and circumferential stress variation in figures 14-17 under four different expansion rates of $13 \%$, $18 \%, 23 \%$ and $28 \%$, respectively. The elastic recovery is the largest in the fourth layer. The circumferential stress variation is the smallest in the fourth layer or the fifth layer. The elastic recovery of each layer is nonlinearly related to

\subsection{Influence of casing elastic recovery on residual stress}

When the expansion cone passes through the area where the expansion has been completed, the casing will have elastic deformation recovery, which will reduce the residual stress of the casing expansion process. It shows the circumferential stress and displacement changes of an element in the casing during $0.043 \mathrm{~s} \sim 0.048 \mathrm{~s}$ when the expansion rate is $18 \%$ in figure 13 . The time when the expansion cone passed through the element with the maximum displacement is $0.043 \mathrm{~s}$, and the time when the expansion process ends is $0.048 \mathrm{~s}$. The radial displacement of the element reduces from $10.03079 \mathrm{~mm}$ to $9.985573 \mathrm{~mm}$ during $0.043 \mathrm{~s} \sim 0.048 \mathrm{~s}$. The variation of elastic recovery is $0.088216 \mathrm{~mm}$. The maximum circumferential stress decreases from 466.348 $\mathrm{MPa}$ to $223.81 \mathrm{MPa}$, and the circumferential stress variation is $242.538 \mathrm{MPa}$.

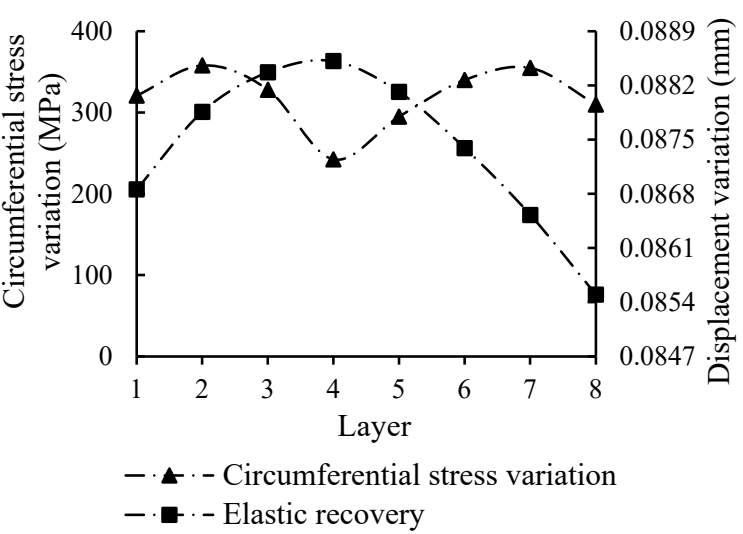

Figure 15. Elastic recovery and circumferential stress variation of $18 \%$ expansion rate.

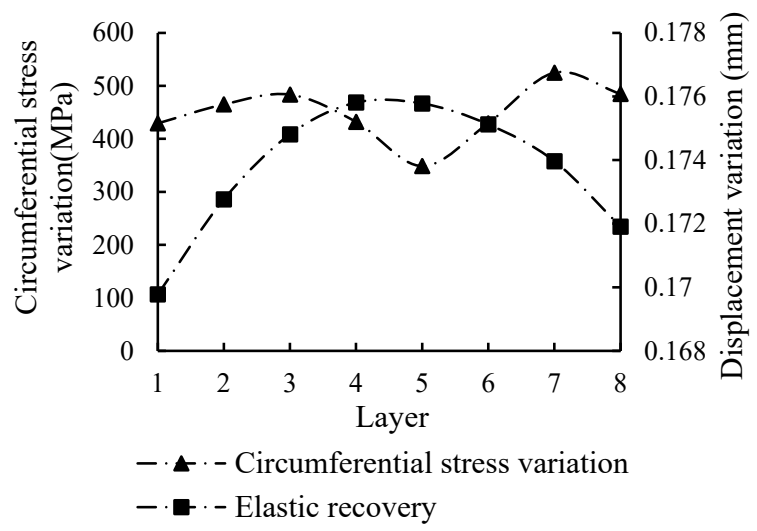

Figure 17. Elastic recovery and circumferential stress variation of $28 \%$ expansion rate.

the stress variation. The elastic recovery of the inner wall and the outer wall of the casing is smaller than other layers, but the circumferential stress variation is greater.

It shows the relationship between the overall average elastic recovery and the circumferential average stress variation after casing expansion under different expansion rates in figure 18 . The greater the average elastic recovery is, the greater the stress variation is. It indicates that more extensive elastic recovery can eliminate a greater degree of residual stress caused by expansion. When the 
expansion rate increases from $13 \%$ to $18 \%$, the variations of the average elastic recovery and stress decreases, which are $0.087393 \mathrm{~mm}$ and $318.6783 \mathrm{MPa}$, respectively. When the expansion rate exceeds $18 \%$, the average elastic recovery and stress variations are linear with the expansion rate. When the expansion rate is $28 \%$, the average elastic recovery and stress variation are the largest, which are $0.173742 \mathrm{~mm}$ and $449.8745 \mathrm{MPa}$, respectively. The greater the elastic recovery after casing expansion is, the more the casing diameter shrinks. Casing shrinkage will reduce the bonding force between the expansion casing and the subsidized casing in the wellbore, resulting in a weakening of the sealing performance between the two casings.

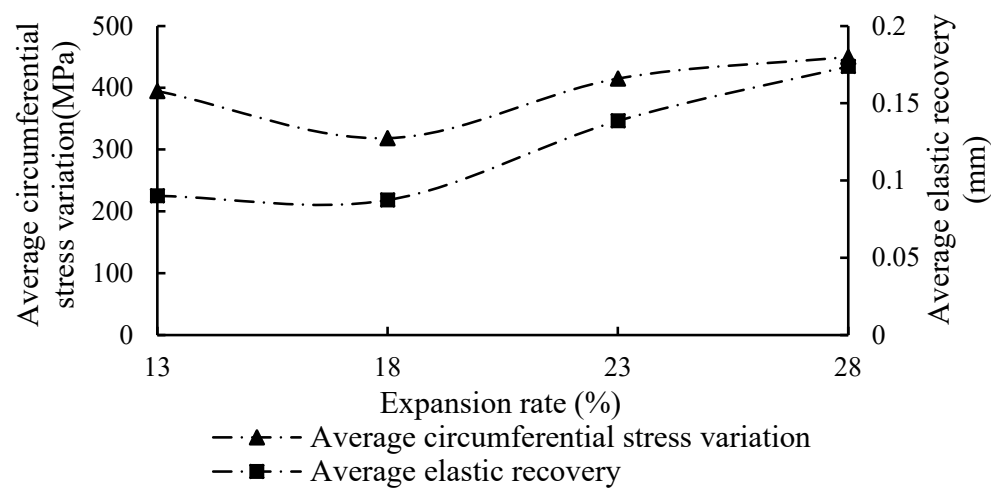

Figure 18. Average elastic recovery and average circumferential stress variation of different expansion rates.

\section{Conclusions}

(1) After casing expansion, residual tensile stress is near the inner wall of the casing, and residual compressive stress is near the outer wall of the casing. The maximum residual tensile stress locates in the middle of the casing thickness direction, and the maximum residual compressive stress locates in the outer wall of the casing.

(2) Due to uneven deformation of casing expansion plasticity, the residual tensile stress and residual compressive stress coexist in the inner wall and end faces of the casing.

(3) The circumferential residual stress after the casing expansion will not increase when the expansion rate increases. When the expansion rate exceeds $18 \%$, with an increase of the expansion rate, the circumferential residual stress has no noticeable change. Selecting the appropriate expansion rate can not only expand the wellbore size but also reduce residual stress to improve the casing collapse resistance. For this material of expansion casing, the expansion rate of $23 \%$ is the most appropriate.

(4) The elastic recovery after casing expansion will reduce the circumferential residual stress, and the elastic recovery is linearly related to the circumferential stress variation. The greater the elastic recovery is, the more the circumferential residual stress decreases. However, the relationship between elastic recovery and expansion rate is nonlinear. The expansion rate increases from $13 \%$ to $18 \%$, but the elastic recovery decreases from $0.09001 \mathrm{~mm}$ to $0.087393 \mathrm{~mm}$.

\section{Acknowledgements}

The authors are grateful for the financial support from the National Natural Science Foundation of China (Nos. 51974251 and 51905426), Key
Research and Development Program of Shaanxi(No.2020KW-015) and CNPC Innovation Found(2019D-5007-0305).

\section{References}

1. Seibi A, Pervez T, Alhiddabi S, et al. Finite Element Modeling of a Solid Tubular Expansion-A Typical Well Engineering Application[J].2005

2. Yongkyu K, Myungho S. Effects of Expanding Methods on Residual Stress of Expansion Transition Area in Steam Generator Tube of Nuclear Power Plants, 2012, 21(4):362-372.

3. Kiptisia W, Namgung I. Analysis of Residual Stresses on the Expanding Transition Zone of Steam Generator Tubes of Apr1400[J]. Journal of Pressure Vessel Technology, 2019.

4. Bouzid A, Zhu L. Effect of Adjacent Tube Expansion on the Residual Contact Pressure of Tube-toTubesheet Joints[J]. American Society of Mechanical Engineers Pressure Vessels \& Piping Division Pvp, 2016:V002T02A016.

5. Bouzid A, et al. A study of neighbouring tube expansion effect on the residual contact pressure of tube-to-tubesheet joints. 2018,

6. Xu B, Zhang Y, Hui W, et al. Application of numerical simulation in the solid expandable tubular repair for casing damaged wells[J]. Petroleum Exploration \& Development,2009,36(5):651-657

7. Zhang J, Han J, et al. Residual stress in expansion casing $[\mathrm{J}]$. Drilling \& Production Technology, 2005(02):18-20+82.

8. Tang $\mathrm{M}$, Jin $\mathrm{Y}$, et al. Residual stress in expansion casing its distribution[J]. Journal of China University of Petroleum, 2008,32(06):125-128.

9. Tang M, Wang L, et al. Mechanical properties of 
expansion casing and residual stress after expansion[J]. Journal of Xi'an Jiao Tong University,2010,44(07):90-94+113.

10. Tang H, Li Q, et al. The effect of yield strength on distribution of residual stress in expandable tube[J]. The Journal of Welded Tube,2011,34(01):9-12+24.

11. Li J. Mechanical properties and residual stress of expansion[J]. The Journal of Modern Technology and Equipment,2016(11):112+114.

12. Zheng H, Yu X, et al. Researches on residual stress of repairing P110 casing based on ABAQUS[J]. World Sci-Tech R\&D, 2015,37(04):333-337.

13. Zhang J, Zhao H. Expansion casing technology for oil and gas wells[M]. Beijing: Petroleum Industry Press, 2015:173-179. 